Meta

Journal des traducteurs

Translators' Journal

\title{
Le terme « Développeur » est-il un néologisme ?
}

\section{Charles Dupont}

Volume 23, numéro 2, juin 1978

URI : https://id.erudit.org/iderudit/002681ar

DOI : https://doi.org/10.7202/002681ar

Aller au sommaire du numéro

Éditeur(s)

Les Presses de l'Université de Montréal

ISSN

0026-0452 (imprimé)

1492-1421 (numérique)

Découvrir la revue

Citer cet article

Dupont, C. (1978). Le terme « Développeur » est-il un néologisme ? Meta, 23(2), 154-158. https://doi.org/10.7202/002681ar d'utilisation que vous pouvez consulter en ligne.

https://apropos.erudit.org/fr/usagers/politique-dutilisation/ 


\section{DROBLIEMES ET SOLUTIONS}

\section{LE TERME « DÉVELOPPEUR » EST-IL UN NÉOLOGISME ?}

On remarque depuis peu que la presse canadienne, écrite et parlée, emploie le vocable «développeur», calqué évidemment sur developer, pour désigner la personne (morale ou physique) qui fait surgir de terre centres commerciaux, groupes de maisons, immeubles, installations touristiques, etc., mais dont le rôle exact reste très obscur. Même s'il ne figure actuellement dans aucun dictionnaire, il est clair que morphologiquement parlant on ne peut rien reprocher au terme "développeur», dérivé par suffixe du verbe développer. Cependant, on ne peut ignorer l'utilisation abusive chez nous des mots DÉvELOPPER et DÉVELOPPEMENT dans la langue des affaires en général et dans le vocabulaire des opérations immobilières (real estate) en particulier, sous l'influence des termes anglais to develop et development qui peuvent, eux, avoir un sens très concret. Ce sont le plus souvent des faux-amis, car en français, du moins jusqu'ici, ces termes ont un sens abstrait ou encore expriment un concept général ou une notion : par exemple, on peut très bien parler du développement d'une idée, de développement social et même industriel ou touristique, mais il s'agit toujours d'une abstraction, d'une orientation ou de planification, et non des travaux pour réaliser ce développement ou leurs résultats. Mais ne nous égarons pas et revenons plutôt, pour l'instant, à developer dont il faut bien connaître la signification pour être en mesure de lui trouver, si possible, un ou des équivalents français.

Le Random House Dictionary définit le mot de la façon suivante :

A person who invests in and develops the urban or suburban potentialities of real estate, esp. by subdividing the land into home sites and then building houses and selling them ${ }^{1}$.

Quant au Webster's Third International, il n'est guère plus explicite :

1.b. A person who develops real estate; often : one that improves and subdivides land and builds and sells residential structures thereon ${ }^{2}$.

1. The Random House Dictionary of the English Language, New York, Random House, 1966, p. 394.

2. Webster's Third New International Dictionary of the English Language, Springfield, G \& C Merriam Company, 1967, p. 200. 
Il est par ailleurs étonnant de ne pas retrouver ce terme dans des dictionnaires spécialisés récents comme le Black's Law Dictionary, l'Economics Dictionary de D. Moffat, le Dictionary of Economics and Business de E.E. Nemmer, The McGraw-Hill Dictionary of Modern Economics ni même dans une monographie intitulée The Developer's Dictionary and Handbook. Pourtant les ouvrages qui traitent de property development (au sens le plus large) font constamment mention du rôle et des obligations du developer, et c'est donc à leurs auteurs qu'il faut s'en remettre pour y voir plus clair.

Les auteurs du livre Design and Planning of Retail System parlent du developer en ces termes :

In order to bring about the new planned shopping centres, a new kind of operator was required, the developer, whose primary role was to make the new centre happen; to assemble the finance, land, tenants and professionals and building skills in such a way that a complete shopping centre would be produced by a certain date and thereafter function in such a way as to provide a satisfactory return on the capital invested. [...] The role is quite distinct from that of the banker, landowner, retailer, architect or contractor, although it could be undertaken by one of these ${ }^{3}$.

Quant à Maurice A. Unger, dans Real Estate Principles \& Practices, il est également très clair, mais en ayant comme point de départ subdivider. Il écrit :

A subdivider is one who buys underdeveloped acreage, divides it into smaller parcels, and sells it. A developer is one who advances the process a step further by building homes on the lots before selling them. Sometimes, in addition to homes, the developer will build a controlled shopping center which provides convenient and accessible shops for the people living in the development. In practice the terms are frequently used interchangeably ${ }^{4}$.

Malgré la dernière remarque de Unger, il ne fait aucun doute que developer est beaucoup plus courant que subdivider, surtout employé lorsqu'il est nécessaire de vraiment distinguer les deux étapes, soit la division des terrains en lots (lotir) d'une part, et la construction comme telle de maisons, d'immeubles ou de centres commerciaux d'autre part. Enfin, Claude Chaline, dans un ouvrage intitulé l'Urbanisme en Grande-Bretagne, définit le vocable developer ainsi :

Désigne ordinairement le promoteur privé responsable d'une opération d'urbanisation dans un but spéculatif ; entre les deux guerres on utilisa l'expression speculative builder pour désigner les créateurs de lotissements bon marché ${ }^{5}$.

PROMOTEUR, voilà donc le terme qui serait l'équivalent français de developer, cet homme-orchestre qui s'occupe de tous les aspects de la mise en valeur d'un fonds de terre (financement, achat des terrains, choix des entrepreneurs, location, etc.) dans un but spéculatif et derrière lequel se cache parfois une banque,

3. David Gosling, Barry Maitland, Design and Planning of Retail Systems, New York, Whitney Library of Design, 1976, p. 28.

4. Maurice A. Unger, Real Estate Principles \& Practices, Cincinnati, South-Western Publishing Co., 5th Edition, 1974, p. 659.

5. Claude Chaline, l'Urbanisme en Grande-Bretagne, Paris, Librairie Armand Colin; Collection $U_{2}, 1972$, p. 200. 
un architecte ou un entrepreneur. Maintenant que le sens de developer est bien circonscrit, voyons si l'équivalent utilisé par Chaline est juste.

D'abord l'Encyclopédie $d u$ bon français dans l'usage contemporain formule sur ce vocable le commentaire suivant :

Ce mot indique la participation effective et active à une entreprise, à la différence d'instigateur qui n'envisage que la pensée initiatrice. Le mot connaît une grande extension de sens depuis quelques années à cause du nouveau système financier en usage dans la construction des immeubles (surtout d'habitations) et de l'importance du promoteur immobilier ${ }^{6}$.

De son côté, le Centre international du droit des affaires (CIDA) définit le mot promoteur ainsi :

Constructeur d'immeubles collectifs qui achète le terrain, finance des emprunts, rédige des actes de vente, gère les bureaux de vente, fait de la publicité, choisit des architectes et des entrepreneurs, fait construire et surveille la gestion des immeubles construits jusqu’à leur achèvement ${ }^{7}$.

Il ne fait plus de doute qu'en ce qui concerne la réalisation d'opérations immobilières dans un but spéculatif, PROMOTEUR (IMMOBILIER) est l'équivalent français de developer. (On trouvera des précisions sur les différents types de promoteurs, leur travail et leur vocabulaire dans l'ouvrage de Jean-François Dhuys voir bibliographie.) Nous pourrions nous arrêter ici, mais nous aurions l'impression de n'avoir donné qu'un élément de solution à un problème plus vaste. Aussi, pour compléter notre recherche terminologique et démontrer la richesse du français dans ce cas, voulons-nous revenir au terme anglais development dont nous décriions l'influence plus tôt.

Dans le sens particulier qui nous intéresse ici, le terme development signifie selon The Developer's Dictionary and Handbook :

1) A tract of land served with utilities and other facilities such as road access.

2) A group of similarly designed houses usually constructed by one builder.

3) A subdivision ${ }^{8}$.

Au Canada on nomme à tort ces réalités « développement ». Le terme exact est LOTISSEMENT, comme le signale le Service de linguistique de Radio-Canada dans ses fiches, car ce mot ne désigne plus aujourd'hui seulement l'action de diviser un terrain en lots (to subdivide), mais aussi le terrain ainsi loti et l'ensemble des habitations qu'on y a construites. Évidemment cet élargissement de l'aire sémantique s'applique aussi à LOTISSEUR (autre équivalent de developer), personne dont le rôle n'est plus uniquement d'acheter des terrains près des villes ou ailleurs et de les munir de l'équipement d'infrastructure nécessaire, mais aussi de procéder à la construction de maisons et parfois même d'usines, d'entrepôts, etc., lorsqu'il s'agit d'une ZONE INDUSTRIELle (industrial park). Dans son accep-

6. P. Dupré, Encyclopédie du bon français dans l'usage contemporain, Paris, Editions de Trévisse, 1972 , tome III, p. 2102.

7. Centre international du droit des affaires, Lexique pratique commercial, Paris, Regif, 1973, p. 324.

8. Koder M. Collison, The Developer's Dictionary and Handbook, Lexington (Mass.), Lexington Books, 1974, p. 35. 
tion première, lotisseur est l'équivalent de subdivider, qu'on a vu plus tôt. Mais cette extension de sens ne s'est pas faite d'elle-même. Elle est principalement due aux lois adoptées en France et ailleurs qui, dans le but de combattre la spéculation foncière, obligent aujourd'hui les lotisseurs à ne plus seulement lotir (dans son acception première) les terrains acquis, mais aussi à les viabiliser [\&De viabilité. Munir un terrain (destiné à la construction) des équipements nécessaires : canalisations d'eau et d'électricité, égouts, voirie, etc. ${ }^{\theta}{ }^{~}$ ] et même dans certains cas à y ériger des constructions avant de pouvoir les revendre. Dans ces cas, le lotisseur se fait, en somme, promoteur. D'un autre point de vue, lorsqu'il ne s'agit pas de construction de logements, mais de travaux d'une plus grande envergure comme la construction d'un aéroport, d'un port ou la mise en valeur de toute une région, development se traduit couramment par AMÉNAGEMENT qui est «l'organisation de l'espace, de manière à mettre en valeur, par des équipements appropriés, les ressources naturelles du lieu et de satisfaire les besoins des populations intéressées ${ }^{10} \%$. L'aménagement (du territoire, urbain, rural, etc.) est une notion relativement nouvelle (début des années 50) qui a donné naissance au terme AMÉNAGEUR qu'on retrouve dans tous les ouvrages sur le sujet et que le dictionnaire les Mots dans le vent (1971) définit ainsi :

Spécialiste de l'aménagement tant dans les domaines de l'urbanisme que sur le plan de la rénovation rurale. L'aménageur doit pouvoir s'intégrer au sein des équipes et des organismes pluridisciplinaires d'aménagement et d'urbanisme ${ }^{11}$.

Il faut ajouter ici que les mêmes auteurs mentionnent par contre AMÉNAGISTE dans l'édition de 1974 du même ouvrage. Ils tirent leur exemple de la revue Forces $\left(\mathrm{n}^{0} 18,1972\right)$ et donnent cette définition :

D'abord spécialiste de l'aménagement des forêts et des fonds d'eau pour la pisciculture. Cette acception s'est étendue à des travaux d'une toute autre ambition. [...] On peut ainsi définir l'aménagiste (angl. developer) comme l'architecte en plein vent de la santé, de la beauté et du respect de la nature, dans un complexe fonctionnel d'un modernisme sans précédent ${ }^{12}$.

Bien qu'aménagiste ait l'avantage de pouvoir s'utiliser tant au masculin qu'au féminin (argument de poids à notre époque), nous croyons qu'aménageur a une bonne longueur d'avance en vue de sa construction par l'usage. De plus, comme la langue n'accepte pas longtemps que deux mots occupent la même aire sémantique, l'un d'eux devra disparaître ou se confiner dans un sens particulier.

Comme on peut s'en rendre compte, la notion d'aménagement a évolué de façon semblable à celle de lotissement et, à cet égard, il ne s'agit plus uniquement (bien que cela ne soit pas exclu) de l'action de préparer les terrains en vue de recevoir des équipments, mais ausși d'y construire effectivement des installations de toutes sortes.

9. Pierre Gilbert, Dictionnaire des mots nouveaux, Paris, Hachette-Tchou, 1976, p. 564.

10. C.I.L.F., la Banque des mots, Paris, Presses universitaires de France, 1971, no 2, p. 205.

11. J. Giraud, P. Samart, J. Riverain, les Mots dans le vent, Paris, Librairie Larousse, 1971, p. 15.

12. Ibid., 1974, p. 13. 
Enfin l'expression MAÎTRE D'EUUVRE est parfois incorrectement utilisée dans le sens de developer, que d'aucuns disent être synonyme de promoteur. Le maître d'œuvre est « celui à qui le maître de l'ouvrage [ou d'ouvrage] confie la conception, l'étude, la direction des travaux et, éventuellement, la coordination des techniciens spécialisés ${ }^{13} »$ ou encore, en droit administratif ou civil, la «personne, entreprise qui est chargée de réaliser l'ouvrage pour le compte du maitre de l'ouvrage. Dans le cas de travail public en régie, maître d'œuvre et maître de l'ouvrage sont confondus ${ }^{14} \gg$. L'équivalent français de developer serait plutôt, on l'aura deviné, le MAîTRE DE L'OUvRAGE qui est «quiconque prend la décision de construire un immeuble, en définit les caractéristiques générales et en gère le risque financier ${ }^{15} »$ ou, en droit administratif, la "personne pour le compte de laquelle un ouvrage, au sens immobilier du mot, est réalisée ${ }^{16} »$.

Pour résumer, nous pouvons donc dire que «développeur» est un calque inutile, puisque nous avons en français au moins quatre termes pour rendre developer, soit PROMOTEUR (IMMOBILIER), LOTISSEUR, AMÉNAGEUR OU AMÉNAGISTE et MAÎTRE DE L'OUVRAGE ou D'OUVRAGE. Il ne s'agit cependant pas de synonymes parfaits. Promoteur est évidemment un générique; il peut désigner aussi bien la personne physique que morale et tant l'entreprise privée que publique, bien qu'employé seul il conserve une connotation de spéculation. Quant à LOTISSEUR et à AMÉNAGEUR, ils peuvent désigner soit celui qui effectue la première partie des travaux de mise en valeur des terrains, soit celui qui procède à l'exécution de l'ensemble du projet; ils se distinguent, toutefois, par le fait que le lotisseur œuvre surtout dans le secteur de l'habitation et l'aménageur dans les grands travaux. Il faut utiliser ces deux mots dans un contexte clair. Enfin, l'expression MAÎTRE DE L'OUVRAGE appartient à un autre registre et, dans une certaine mesure, à la langue juridique. À cause de cela et de la confusion qui existe souvent avec maître d'œuvre, il est préférable de la réserver aux textes spécialisés.

Charles Dupont

\section{TABLEAU DE SYNTHĖSE}

developer

subdivider

\author{
promoteur (immobilier) - générique \\ lotisseur - spécifique \\ aménageur - spécifique \\ maître de louvrage - générique, mais spécialisé \\ lotisseur (sens restreint)
}

\section{SOURCES CONSULTÉES}

BRISSY, Yves, les Villes nouvelles, Paris, Éditions Berger-Levrault, 1974, 247 p.

JAMOIS, Jean, les Zones à urbaniser par priorité (ZUP), Paris, Éditions Berger-Levrault, $1968,251 \mathrm{p}$.

13. C.I.L.F., op. cit., 1974, no $^{\circ}$, p. 190.

14. Raymond Guillien, Jean Vincent, Lexique de termes juridiques, Paris, Dalloz, 20 édition, 1972 , p. 212.

15. Jean-François Dhuys, les Promoteurs, Paris, Editions du Seuil, 1975, p. 13.

16. Raymond Guillien, op. cit., p. 212. 